\title{
$\mathrm{PPG} \operatorname{LinC}(10$
}

\section{SOBRE A MORFOSSINTAXE DA DETERMINAÇÃO NOMINAL DOS DPs EM PORTUGUÊS}

\author{
ABOUT MORPHOSYNTAX OF NOMINAL \\ DETERMINATION IN BRAZILIAN \\ PORTUGUESE NOUN PHRASES
}

Fernanda de Oliveira Cerqueira ${ }^{1}$ Educação básica - Rede privada

Victor Cavalcanti Mariano ${ }^{2}$ Instituto Federal da Paraíba

Resumo: Considerando que, em uma perspectiva minimalista (CHOMSKY, 1995 e posteriores), a derivação sintática opera com base na valoração de traços formais (CHOMSKY, 1995, 2001, 2005; FRAMPTON; GUTMANN, 2000; BÉJAR, 2003, 2008; CARVALHO, 2008, 2012, 2017), o presente Squib tem por objetivo apresentar o mapeamento dos comportamentos morfossintático e semântico dos sintagmas nominais - DPs (ABNEY, 1987), em Português Brasileiro, a fim de identificar um caminho para a sua composição de traços, observando a pertinência dos traços [Definite] e [Specific], os quais parecem relevantes para derivação e interpretação dos nominais nessa língua (CARVALHO, 2008, 2017; MARIANO, 2012, 2018; CERQUEIRA; CARVALHO, 2018, 2020; CERQUEIRA, 2019a, 2019b, 2020).

Palavras-Chave: Sintagma Nominal; Morfossintaxe; Semântica; Traços; Determinação.

1 f.cerqueira@ufba.br

2 victor.mariano@ifpb.edu.br 
Abstract: Considering that, in a minimalist perspective (CHOMSKY, 1995 and later), syntactic derivation operates based on the valuation of formal features (CHOMSKY, 1995, 2001, 2005; FRAMPTON; GUTMANN, 2000; BÉJAR, 2003, 2008; CARVALHO, 2008 , 2012, 2017), the present Squib aims to present the mapping of the morphosyntactic and semantic behaviors of noun phrases -DPS (ABNEY, 1987), in Brazilian Portuguese, in order to identify a path for its composition of features, observing the pertinence of the [Definite] and [Specific] features, which seem relevant to the derivation of nominals in this language (CARVALHO, 2008, 2017; MARIANO, 2012, 2018; CERQUEIRA; CARVALHO, 2018, 2020; CERQUEIRA, 2019a, 2019b,2020).

Keywords: Noun Phrase; Morphosyntax; Semantic; Features, Determination.

\section{INTRODUÇÃO}

Desde o modelo padrão, da Teoria Gerativa, delineado em torno da busca por adequação descritiva de tipos de gramáticas possíveis (CHOMSKY, 1965), trabalhos como Postal (1966) e Ross (1967) já discutiam a sintaxe e a semântica de determinantes. Porém, a partir de novas evidências e sugestões teóricas, em termos de projeção sintática, apresentadas no modelo de Regência e Ligação (CHOMSKY, 1981, 1986b), novos desafios têm sido colocados para a linguística formal no que tange ao campo da categoria dos nominais. Isso porque, principalmente desde Abney (1987), os chamados sintagmas nominais passaram a ser entendidos como parte de uma projeção sintática mais complexa, a fim de poder dar conta de suas possibilidades de representações morfossintáticas.

Em seu texto, Abney apresenta o que ficou conhecido como hipótese-DP3: para o autor, há correlação entre a estrutura morfossintática dos nominais e da sentença, o que o leva a propor uma projeção do núcleo determinante, o DP. Desse modo, o DP, assim como o IP e o CP para o verbo, é uma projeção ${ }^{4}$ que apresenta informações funcionais e desempenha o papel de estabelecer as

\footnotetext{
3 Siglas apresentadas no texto: $\mathrm{DP}=$ Determiner phrase ou sintagma determinante; $\mathrm{IP}=$ Infelction phrase, do inglês; $\mathrm{CP}=$ Complementizer phrase, do inglês; e $\mathrm{PP}=$ Prepositional phrase,

4 Considerando que discutir aspectos mais cartográficos acerca da estrutura do DP não é intuito do presente trabalho, para tanto, ver Ghomeshi, Paul e Wiltschko (2009), Sedrins (2009) e Cyrino e Espinal (2015).
} 
relações gramaticais nas línguas, como concordância e referência. Sendo assim, a categoria DP dispõe não só de propriedades sintáticas, como também de propriedades semânticas, as quais lhes são próprias, como pode-se observar em (1).

(1) a. [DPO menino] foi para escola.

b. [DP Esses meninos] foram para escola.

c. [DP Ø Menino] foi para escola.

d. * [DP O Meninos] foram para escola.

e. [DP Um belo menino alegre] foi para escola.

f. [DP Todo menino] vai para escola 5 .

Em (1), é possível perceber que há diferentes possibilidades de realização do DP. Em todos os casos, embora os sintagmas determinantes estejam no domínio de DP, apresentam leituras distintas no que concerne à determinação nominal, evidenciando que outro fator, além de sua projeção, parece reger seu comportamento.

Com o advento do Programa Minimalista, (CHOMSKY, 1995 e posteriores), compreende-se que a derivação sintática opera apenas com a valoração de traços formais (FRAMPTON; GUTMANN, 2000; CHOMSKY, 2005), isto é, aqueles imprescindíveis para o licenciamento sentença. Deste modo, abrese possibilidade para a concepção de traço formal como elemento formativo (ADGER; SVENONIUS, 2010; CARVALHO, 2012). Sob essa ótica, alguns traços tradicionalmente observados como interpretáveis, dada à sua natureza léxicosemântica, passam a ser tratados como formais, à proporção que passam a interferir no licenciamento de fenômenos sintáticos.

Nessa direção, Carvalho (2008) propõe que o traço [D], proposto por Chomsky (1995), equivalente ao antigo traço EPP (CHOMSKY, 1995), seja o traço

\footnotetext{
5 Sabe-se que, em português, traços de tempo e de aspecto interferem no escopo de quantificação existencial da sentença (DUARTE; OLIVEIRA, 2006) e, consequentemente, na genericidade do DP (CARLSON, 1989; CARLSON; PELLETIER, 1995; CHIERCHIA, 1995; MÜLLER, 2000; OLIVEIRA; MEZARI, 2012).
} 
responsável pela codificação de DPs, à medida que esse traço representa uma característica de sintagmas determinantes em geral, assim,

[[D] é o] traço corresponde ao traço [N] e caracteriza nominais. A presença deste traço caracteriza o nominal como sendo um argumento, como defendem alguns autores (DÉCHAINE; WILTSCHKO, 2002). Este traço também domina a projeção que compreende traços como [DEFINITE] e [SPECIFIC] (CARVALHO, 2008, p. 80).

Portanto, o traço [D], inerente às expressões nominais, aos pronomes e às anáforas (CHOMSKY; LASNIK, 1993; CHOMSKY, 1995), parece tanto ser um requerimento sintático para a distribuição de DPs, quanto de sua contraparte léxico-semântica. Em vista disso, assumimos com Cerqueira e Carvalho (2018, 2020) e Cerqueira (2019a, 2019b, 2020) que tais propriedades estruturais e referenciais sejam categorizadas como determinação nominal.

Nessa ótica, a determinação nominal é uma propriedade das línguas humanas, própria de determinantes, pois “[...] a determinação está predominantemente associada à função referencial, isto é, à introdução (ou à retomada anafórica) de entidades [...]" (MÓIA, 2016, p. 313). Logo, os determinantes possuem características próprias não só sintáticas, como também semânticas. Assim, Abraham, Stark e Leiss (2007) propõem que

determinação [...] pode ser vista como a marcação explícita de nominais para fornecer informações sobre a forma como o predicado nominal deve ser mapeado em diferentes tipos (escolha de conjuntos bem como texto) de referência (no sentido de Seiler (1978) 'noção de percepção de realidade' (ABRAHAM; STARK; LEISS, 2007, p. 2, tradução nossa').

Desta maneira, “[...] outras características tais como estatuto temático ou definitude/especificidade em contextos definidos e indefinidos podem entrar em

\footnotetext{
6 No original: "[d]etermination [...] can be seen as the explicit marking of nominals in order to provide information about the way the nominal predicate is to be mapped onto different sorts of (choices of sets of as well as text) referents (in the sense of Seiler's (1978) notion of 'perception of reality') (ABRAHAM, et al., 2007, p. 2).
} 
jogo de maneira adequada ou exclusiva" (ABRAHAM et al., 2007, p. 2, tradução nossa7). Com efeito, a determinação nominal é estabelecida na medida em que um DP apresenta as leituras de definitude e/ou de especificidade. Entretanto, ainda que a determinação nominal seja um fenômeno universal, sua manifestação varia nas línguas humanas, de modo que os possíveis comportamentos morfofonológicos podem ou não refletir padrões sintáticos, como argumenta Correia (2000):

[...] línguas como o português, o francês, o italiano e o inglês utilizam diferentes classes de determinantes (artigos definidos, indefinidos, determinantes nulos, partitivo, numerais, possessivos, demonstrativos ...) como marcas de determinação nominal. As línguas sem artigo, como o japonês, o coreano ou o mandarim, evidenciam a determinação nominal através de classificadores, ou através da ordem das palavras, ou, ainda, através de processos morfológicos como a sufixação ou a prefixação" (CORREIA, 2000, p. 328).

Destarte, o presente Squib tem como objetivo realizar o mapeamento dos contextos morfossintático e semântico dos DPs, em Português Brasileiro (PB), com intuito de apresentar caminhos para sua composição de traços- $\varphi$ (HARLEY; RITTER, 2002; BÉJAR, 2003, 2008; CARVALHO, 2008, 2017), observando a pertinência dos traços [Definite] e [Specific], os quais parecem relevantes (CARVALHO, 2008, 2017; MARIANO, 2012, 2018; CERQUEIRA, CARVALHO, 2018, 2020; CERQUEIRA, 2019a, 2019b, 2020).

\footnotetext{
7 No original: "[o]ther characteristics such as thematic or definite/specific status in definite and indefinite contexts may come into play as well or exclusively" (ABRAHAM; STARK; LEISS, 2007, p. 2).
} 


\section{O COMPORTAMENTO MORFOSSINTÁTICO E SEMÂNTICO DE DPS}

Nesta seção, apresentaremos os dados a fim de identificar pistas sobre a natureza do DP, por meio do cruzamento de seu comportamento morfossintático com suas leituras semânticas. Outrossim, assumimos, com Abraham, Stark e Leiss (2007), Cerqueira e Carvalho (2018, 2020) e Cerqueira (2019a, 2019b, 2020), que determinação nominal, [D], seja uma propriedade estrutural e referencial de DPs, consequentemente, responsável pelos comportamentos sintático e léxicosemântico dos itens nessa projeção.

No que concerne aos traços de definitude e de especificidade, os quais acarretam [D]', assumimos, com base nos trabalhos supracitados, que: a. a leitura identificável (o referente de uma expressão nominal é acessível tanto ao falante, quanto ao ouvinte) é reflexo da presença de definitude na composição do DP (LYONS, 1999; ABBOTT, 2010; HERTZENBERG, 2015), e b. a leitura particularizada (a expressão nominal corresponde à totalidade dos possíveis referentes) é reflexo da presença de especificidade na composição do DP (PARTEE, 1972, 2004; ABBOTT, 2010), uma vez que

[...] para Donellan (1966), Lyons (1999) e Abbott (2010), para Cowper e Hall (2002) os efeitos semânticos de definitude podem ser: a) identificabilidade ou unicidade, pelo ouvinte; ou b) identificabilidade e unicidade, pelo ouvinte. Todavia, a possibilidade de intercorrência desses efeitos implica que: i) cada uma dessas leituras é efeito de um elemento primitivo; e ii) a presença de ambas é efeito de um elemento mais abrangente, ao qual definitude e especificidade estão subjacentes. Por conseguinte, assumo, com Hertzenberg (2015), que identificabilidade é efeito de definitude; com Partee (2004), que unicidade é efeito de especificidade; e, com Cerqueira e Carvalho (2018a, 2018b), que identificabilidade e unicidade juntas representam efeito de determinação nominal (CERQUEIRA, 2019a, p. 94).

8 Os dados utilizados neste trabalho são de introspecção, haja vista que o falante nativo de uma língua humana qualquer é competente em sua Língua-I, sistema da mente/cérebro do falante nativo que lhe permite conhecer sua língua materna (CHOMSKY, 1986a).

9 A discussão em torno do traço [D], bem como da geometria de traços, pode ser verificada em Carvalho (2008). 
Além das leituras anteriormente expostas, consideramos que genericidade seja a propriedade de um nominal fazer referência a uma categoria com o sentido de tipo, espécie, gênero ou grupo (CARLSON, 1989, CARLSON; PELLETIER, 1995; KRIFKA et al., 1995; CHIERCHIA, 1995; SARAIVA, 1997; OLIVEIRA, 2001). Não obstante, assumimos que o DP genérico não dispõe de leituras ${ }^{10}$ identificável e particularizada, devido à ausência de definitude e de especificidade, respectivamente. Assim sendo, as subseções a seguir serão destinadas à apresentação da morfossintaxe de DPs em função de: 1.1 Determinação nominal; 1.2 Definitude; 1.3 Especificidade; e 1.4 Genericidade.

\subsection{Determinação nominal}

De acordo com Abraham, Stark e Leiss (2007), DPs determinados são aqueles em que se verifica maior marcação/especificação e cuja natureza, nos termos aqui propostos, se dá em função das leituras identificável e particularizada, oriundas, respectivamente, da presença de definitude e de especificidade na composição interna ${ }^{11}$ desse sintagma.

(2) a. Maria saiu cedo.

b. Eu moro em Salvador.

(3) a. Faz tempo que espero a conclusão dessa obra, porque a construção simplesmente não acaba.

b. Amanhã, haverá uma exposição de grafite e com certeza o evento vai bombar.

(4) a. Essa universidade tem ótimos professores.

b. Aquele livro chegou hoje na biblioteca.

(5) a. Adotei meus gatos.

b. Seus gatos vivem aqui em casa.

10 Em linhas gerais, a leitura identificável corresponde à definitude e a leitura particularizada corresponde à especificidade. Contudo, essa discussão será melhor apresentada adiante.

11 Compreende-se composição interna como a hierarquia de traços formais do DP a ser valorada no curso da derivação. 
c. Os gatos dele vivem aqui em casa.

(6) a. A mãe de Maria sempre faz doces para nós.

b. Comprei a sandália da Peppa Pig para você.

(7) a. A democratização das mídias é uma demanda urgente.

b. Pedro, estou até hoje aguardando a conclusão do trabalho.

Em (2), há dados de DPs determinados com nome próprio, os quais, segundo Strawson (1950), Donellan (1966), Lyons (1977), Lyons (1999), apresentam referência plena, portanto, leituras identificável e particular. Em (3), há DPs com realização de artigo e, em (4), com pronomes demonstrativos. Em (5), há DPs com leitura de posse, sendo, em (5a-b), com pronomes possessivos canônicos, e, em (5c), com possessivo não canônico (FONSECA, 2019). Em (6), há DPs com adjunção de PP, com destaque para (6a), na qual há DP com leitura de posse inalienável, em oposição a (5c). Por fim, em (7), há DPs complementados por PPs.

Assim, os dados acima ilustram diferentes comportamentos da determinação nominal em $\mathrm{PB}$, tendo em vista as diferentes configurações morfossintáticas desses DPs, ainda que em todos estes casos haja leitura identificável e particular, ocasionadas pela presença dos traços de definitude e de especificidade, respectivamente, em sua composição. Tal fato corrobora com a proposta de Correia (2000) na qual a autora apresenta evidências de que a determinação nominal possui diferentes manifestações morfofonológicas nas línguas naturais, ainda que sua contraparte semântica seja definida e/ou específica (ABRAHAM et al., 2007; CERQUEIRA, 2019a, 2019b, 2020).

\subsection{Definitude}

Definitude é, tradicionalmente, tratada como uma propriedade do artigo definido (HAWKINS, 1978; LYONS, 1999), reforçando a ideia de que essa propriedade é estritamente morfossintática, o que parece não proceder, conforme 
defendem os trabalhos de Cowper e Hall (2002) e Carvalho (2008), os quais argumentam em função de que a presença de artigo em nome definido é apenas reflexo de sua composição léxico-sintática do DP.

(8) a. O pessoal faz festa por tudo.

b. Mesmo em pandemia, a galera fica na porta.

c. As manas estão articuladas.

Nas sentenças em (8), há DPs apenas com leitura identificável, uma vez que, embora não seja possível precisar quem "faz festa por tudo", quem "fica na porta", ou quais minas "estão articuladas", seu contexto assegura uma noção de familiaridade (CHRISTOPHERSEN, 1939; HEIM, 1982; LYONS, 1999) com estes referentes, de modo que suas leituras parecem identificáveis. Ou seja, aparentemente, é a composição de traços desses nominais, com efeito em sua leitura identificável, o que lhes confere caráter definido. Em contrapartida, os dados em (9) não apresentam especificidade, haja vista que não há leitura particularizada, conforme será discutido na próxima subseção.

\subsection{Especificidade}

De acordo com Partee (1972, 2004), uma entidade única precisa ser particularizada com base em restrições, por meio das quais se torna específica. Nessa direção, Cowper e Hall (2002, p. 3) defendem que a presença do traço de especificidade, na composição do DP, implica em leitura particularizada, como um tipo de quantificação cardinal. Deste modo, ao invés de quantificar sobre indivíduos existenciais ou universais, a especificidade quantifica sobre um indivíduo particular.

Esse comportamento pode ser observado nos dados em (9), cuja leitura particularizada decorre do numeral "um" e da relativa "que gosta de anime", os 
quais especificam os DPs. Diante disso, não há, nos dados em (9), leitura identificável.

(9) a. Quando um país não investe em educação, está fadado ao fracasso.

b. Uma menina que gosta de anime vai ao Bonodori.

Em (9), a especificação de DP ocorre devido ao seu comportamento de entidade única favorecido pela leitura de cardinalidade do numeral, a qual pode ser associada à indefinição.

\subsection{Genericidade}

Quanto aos nominais com leitura genérica ${ }^{12}$, observa-se que esses sintagmas, como dito acima, não apresentam nem leitura particularizada, nem leitura identificável, aparentemente, devido à ausência dos traços de definitude e de especificidade, em sua composição de traços no léxico.

Tradicionalmente ${ }^{13}$, assume-se que os artigos são responsáveis pela realização morfofonológica de definitude e de especificidade, já que, no caso dos nominais genéricos, a total ausência de determinação nominal é atribuída à ausência de determinante. Em vista disso, acredita-se que os substantivos sejam nus (sem determinantes), uma vez que esses nominais se referem a uma classe, como apontado anteriormente. Assim, Carlson (1989) argumenta que os bare plurals (nomes nus plurais) do inglês funcionam como nomes próprios de espécie,

12 Embora a propriedade semântica de genericidade não seja particularidade de DPs ou NPs, a depender da abordagem, no presente trabalho a discussão centra-se no sintagma determinante.

13 De acordo com Correia (2000), acerca das realizações de determinação nominal, e, mais recentemente, Carvalho (2020, 2021), no que tange ao gênero gramatical, há problemas com generalizações assertivas sobre a universalidade das línguas humanas considerando estritamente a morfossintaxe de línguas indo-europeias, pois além de incorrer no fenômeno do epistemicídio (CARNEIRO, 2005), ainda reduz a compreensão da GU ao padrão verificado em línguas de colonização (ABOH, 2020). 
indicando a generalização. Tais construções podem ser exemplificadas, em PB, pela sentença em (10):

(10) a. Elefantes têm tromba.

A fim de comprovar a hipótese de que bare plurals são nomes de espécies, Carlson estabelece uma comparação entre as diferentes interpretações que esses nominais podem ter: a particularizada (que, antes da hipótese carlsoniana, era compreendida como indefinido plural) e a genérica (como aqui apresentada). $\mathrm{O}$ autor argumenta, em sua análise, que essas duas leituras não são acarretadas por uma ambiguidade dos nominais nus (ou, nessa discussão, pelos seus traços formais), mas do predicado em que o nome é realizado.

Deste modo, para o autor, não devem existir diferentes tipos de plurais nus, mas somente um tipo, que denota espécie (ou seja, não possui leitura identificável ou particularizada) e que assume diferentes leituras a depender da sentença em que esteja (predicados de indivíduos - leitura genérica vs. predicados de estágio - leitura particularizada).

Entretanto, não é incomum, nas línguas naturais, que os nominais genéricos estejam em estruturas com artigos realizados, tanto definidos, quanto indefinidos, como em PB, em (11).

(11) a. Um elefante tem tromba.

b. O elefante tem tromba.

c. Os elefantes têm tromba.

O exemplo em (11a) é lido como: para ser um elefante, é preciso ter tromba. Em (11b), a leitura é de que todo e qualquer elefante possui tromba, como em (11c). Esses dados revelam que os artigos, nesses casos, não refletem as leituras identificável, nem particularizada, uma vez que, aparentemente, os traços de definitude e de especificidade estão subespecificados, na composição destes DPs. 
Outro aspecto que contribui com as propostas de Correia (2000) e Cerqueira (2019a), haja vista que além dos dados apresentados, na seção anterior, de genéricos com artigos, existem outros exemplos, em PB e em outras línguas, considerando que as leituras identificável e particularizada são observadas em nominais completamente nus, como em dados de comunidades quilombolas brasileiras, como Helvécia (MARIANO, 2012, 2018; RIBEIRO; CYRINO, 2010).

Nessa perspectiva, a respeito do comportamento da realização de nominais, em PB, observa-se a ocorrência de nomes nus singulares em posições argumentais, diferenciando-o de muitas línguas românicas e de línguas germânicas (SCHIMITT; MUNN, 1999). Em línguas como essas, a realização de nomes nus é limitada, sendo incomum sua ocorrência, quando singulares, em posições argumentais, com leituras particularizada ou genérica. Assim, os exemplos abaixo ilustram a diferença entre o PB e o Português Europeu.

(12) Português brasileiro

a. Cachorro gosta de gente. (Genérico)

b. Mulher esteve discutindo política (Existencial)

(13) Português europeu

a. * Cachorro gosta de gente. (Genérico)

b. *Mulher esteve discutindo política (Existencial)

(SCHIMITT; MUNN, 1999, p. 7-8)

Além disso, Kabatek (2007) revela que, no PB, também há contextos em que ocorrem os nomes nus singulares com leitura identificável e particulariza. Dessa forma, o autor argumenta que contextos, como os de manchetes de jornais, favorecem a realização dos nominais nus singulares determinados, além de evidenciar sua produtividade, como pode-se observar em (14).

(14) a. Bolsa cai e dólar dispara com alta de petróleo (O Estado de S. Paulo, 19.9.2000)

b. Bicicleta não polui (propaganda) 
c. Bicicleta ia se arrebentar (Banco de dados interacionais - Roncarati UFRJ)

(KABATEK, 2007, p. 31-38)

Tais dados revelam que a referência dos nominais parece ser condicionada pela presença dos traços de definitude e de especificidade, mas não necessariamente à realização de morfofonológica do determinante. Nesse sentido, argumenta-se que a determinação nominal seja um fenômeno universal, mas sua realização morfofonológica seria variável nas línguas naturais (seja através da presença de artigo, seja através de relações nas sentenças, ou ainda de honoríficos, por exemplo). Deste modo, a leitura léxico-semântica e a distribuição sintática dos DPs parecem estar relacionadas à sua composição de traços e não à sua morfologia (CORREIA, 2000; ABRAHAM, et al., 2007; CERQUEIRA, 2019a, 2019b, 2020; CERQUEIRA; CARVALHO, 2020).

\section{CONCLUSÕES}

No presente artigo, apresentamos a descrição do comportamento morfossintático do $\mathrm{DP}$, em $\mathrm{PB}$, em função das leituras léxico-semânticas de identificabilidade e de particularidade, decorrentes da presença dos traços de definitude e de especificidade, respectivamente, na composição do sintagma determinante.

À vista disso, observamos que aparentemente não há correlação precisa entre a morfossintaxe do DP e sua semântica, conforme discussão na seção anterior.

Logo, considerando que determinação nominal é um fenômeno universal cuja realização varia nas línguas humanas, ao passo que seus comportamentos morfofonológicos podem ou não refletir padrões sintáticos (CORREIA, 2000; ABRAHAM, et al., 2007), acreditamos que a valoração dos traços de [Definite] e 
[Specific], na derivação, possa ser um caminho para a compreensão deste fenômeno, tendo em vista que esses traços parecem relevantes para a distribuição e para referência do DP, como apontam os trabalhos de Carvalho (2008, 2017); Mariano (2012, 2018), Cerqueira (2015, 2019a, 2019b, 2020), Medeiros Júnior (2017), Cerqueira e Carvalho (2018, 2020) e Fonseca (2019).

\section{REFERÊNCIAS}

ABBOTT, B. Reference. New York: Oxford University Press, 2010.

ABOH, E. O. Lessons From Neuro-(a)-Typical Brains: Universal Multilingualism, CodeMixing, Recombination, and Executive Functions. Frontiers in Psychology, v. 11, artigo 488, 2020.

ABRAHAN, W.; STARK, E.; LEISS, E. Introduction. In: STARK, E.; LEISS, E.; ABRAHAM, W. (Eds.). Nominal Determination: Typology, context constraints, and historical emergence. Philadelphia: John Benjamins B. V., 2007, p. 1-21.

ABNEY, S. The English Noun Phrase in its sentencial aspects, 1987. 363 f. Tese. (Doutorado) MIT, Cambridge.

ADGER, D.; SVENONIUS, P. Features in minimalist syntax. In: BOECKX, C. The Oxford Handbook of Linguistic Minimalism. New York: Oxford University Press, 2010.

BÉJAR, S. Phi-syntax: a theory of agreement. 2003. 214 f. Tese. (Doutorado em Linguística) University of Toronto, Ontário.

Conditions on phi-agree. In: HARBOUR, D.; ADGER, D.; BÉJAR, S. (Orgs.). Phi Theory: Phi-Features across modules and interfaces. New York: Oxford University Press, 2008, p. 130-154.

CARLSON, G. The Semantic Composition of English Generic Sentences. In: CHIERCHIA, G; PARTEE, B.; TURNER, R. (Orgs.) Properties, Types and Meaning, v. 2: Semantic Issues. Dordrecht: Kluwer, 1989, p. 167-.191.

1995.

; PELLETIER, F. J. The Generic Book. Chicago - London: The University of Chicago Press,

CARNEIRO, A. S. A construção do outro como não-ser como fundamento do ser. 2005, $339 \mathrm{f}$. Tese. (Doutorado em Filosofia) São Paulo, USP, 2005.

CARVALHO, D. S. A Estrutura interna dos pronomes pessoais em português brasileiro, 2008. $155 \mathrm{f}$. Tese. (Doutorado em Letras e Linguística) Faculdade de Letras, UFAL, Maceió.

. Traços. In: FERRARI NETO, J.; SILVA, C.R.T. (Orgs.). Programa Minimalista em Foco:

princípios e debates. 1 ed. Curitiba: Editora CRV, 2012, v. 1, p. 113-132. 
. Morfossintaxe de caso e o sincretismo pronominal no português. In: CARVALHO, D. (Org.). Traços-phi: contribuições para a compreensão da gramática do português. Salvador: EDUFBA, 2017, p. 169-188.

. As genitálias da gramática. Revista da Abralin, v. 19, n.1, 2020, p. 1-21.

A domesticação da gramática de gênero. Campinas: Pontes, 2021.

CERQUEIRA, F. O. A sintaxe do pronome acusativo de terceira pessoa no português brasileiro, 2015. 92 f. Dissertação. (Mestrado em Língua e Cultura) Instituto de Letras, UFBA, Salvador.

. O pronome pleno de terceira pessoa: estrutura interna e relações referenciais. 2019a. 152f. Tese. (Doutorado em Língua e Cultura) Instituto de Letras, UFBA, Salvador.

A arbitrariedade de terceira pessoa no português brasileiro. In: CARRILHO, E.; MARTINS, A. M.; PEREIRA, S. (Orgs.). Estudos Linguísticos e Filológicos oferecidos a Ivo Castro, Lisboa: Centro de Linguística da Universidade de Lisboa, 2019b, p. 447-508.

. Pronomes pessoais: participante e determinação como componentes de referência. Estudos interdisciplinares da linguagem, v. 01. Campina Grande: Realize Editora, 2020. p. 95-114.

CERQUEIRA, F. O; CARVALHO, D. O comportamento sintático-semântico do pronome pleno de terceira pessoa no português brasileiro. Dossiê ANPOLL - 30 anos do Grupo de Trabalho em Teoria da Gramática, n.46, v.1, p. 83-111, Florianópolis, 2018.

- A A configuração de referência nos pronomes de terceira pessoa em português. Revista do GELNE. Dossiê Morfologia, n. 22, n2, 2020, p. 321-335.

CHIERCHIA, G. Individual-Level Predicates as Inherent Generics. In: CARLSON, G.; PELLETIER, F. J. (Orgs.). The Generic Book. Chicago: The Universit of Chicago Press, 1995, p. 176223.

CHOMSKY, N. Aspects of the Theory of Syntax. Cambridge, Massachusetts: MIT Press, 1965. . Lectures on Government and Binding. Dordrecht: Foris, 1981.

. Conhecimento da língua: sua natureza, origem e uso. Tradução de Eduardo Raposo. Lisboa: Caminho, 1986a.

. Barriers: Linguistic Inquiry. 13. Cambridge: MIT Press, 1986b.

. Programa Minimalista. Tradução de Eduardo Raposo. Lisboa: Caminho, 1995.

. Derivation by phase. In: KENSTOWICZ, M.; HALE, K. (Orgs). A life in Language. Cambridge, Ma.: MIT Press, 2001.

. On phases. Ms., 2005.

. LASNIK, H. The theory of principles and parameters. In: Syntax: an international handbook of contemporary research. von STECHOW, J. J. A., STERNEFELD, W.; VENNEMANN, T. (Eds.). Berlin: De Gruyter, 1993, p. 506-569.

CHRISTOPHERSEN, P. The articles: a study of their theory and use in English. Copenhagen: Einar Munksgaard, 1939.

CORREIA, C. N. Determinação Nominal. In: Revista da Faculdade de Ciências Sociais e Humanas, Lisboa: Edições Colibri, 2000, n.13, p. 327-338. 
COWPER, E.; HALL, D. C. The syntactic manifestation of nominal feature geometry. In: Proceedings of the 2002 Annual Conference of the Canadian Linguistic Association. Montréal: Cahiers Linguistiques de l'UQAM, 2001, p. 55-66.

CYRINO, S.; ESPINAL, M.T. 2015. On the structure of bare nominals in Brazilian Portuguese. In: SMITH, J.; IHSANE, T. (Eds). Romance Linguistics 2012: Selected papers from the 42nd Linguistic Symposium on Romance Languages (LSRL), Cedar City, Utah, 20-22 April 2012. Amsterdam: John Benjamins, 2015, p. 215-228.

DÉCHAINE, R. M; WITSCHKO, M. Decomposing Pronouns. Linguistic Inquiry, v. 33, n.3, 2002, p. 409-442.

DONELLAN, K. Reference and definite descriptions. Philosophical Review, 1966, n 77, p. 281-304.

DUARTE, I.; OLIVEIRA, F. Referência Nominal. In: MATEUS, M. H. M. et al. Gramática da Língua Portuguesa. Lisboa: Caminho, 2006, p. 211-243.

FRAMPTON, J.; GUTMANN, S. Agreement is Feature Sharing. Ms.: Northeastern University, 2000.

FONSECA, R. M. O comportamento morfossintático dos possessivos canônicos e não canônicos de terceira pessoa no Português Brasileiro. 2019. 89 f. Dissertação. (Mestrado em Língua e Cultura) Instituto de Letras, UFBA, Salvador.

GHOMESHI, J.; PAUL, I.; WILTSCHKO, M. Determiners: universal and variation. Amsterdam: John Benjamins B. V., 2009.

HARLEY, H.; RITTER, E. Person and number in pronouns: a feature-geometric analysis. Language, 2002, v. 78. p. 482-526.

HAWKINS, J. Definiteness and indefiniteness: a study in reference and grammaticality predicaction. London: Croom Helm, 1978.

HEIM, I. The semantics of definite and indefinite noun phrases. Graduate Linguistics Students Ass., University of Massachusetts Mass: Amherst, 1982.

HERTZENBERG, M. J. B. Third Person Reference in Late Latin: demonstratives, definite articles, and personal pronouns in the Itinerarium Egeriae. Library of Congress Cataloging-inPublication Data. De Gruyter Mouton: Berlin/Boston, 2015.

KABATEK, J. Existe um ciclo de gramaticalização do artigo na romania?. In: RAMOS, J.; ALKMIM, M. (Org.). Para a história do português brasileiro: estudos sobre mudança lingüística e história social. 1 ed. Belo Horizonte: Editora FALE/UFMG, 2007, p. 13-51.

KRIFKA, M.; PELLETIER, F.; CARLSON, G. ter MEULEN, A.; CHIERCHIA, G.; GODEHARD, L. Genericity: an introduction. In: CARLSON, G.; PELLETIER, F. J. (orgs.). The Generic Book. Chicago: The Universit of Chicago Press, 1995, p. 1-124.

LYONS, C. Definiteness. Cambridge: University Press, 1999.

LYONS, J. Semantics, 1 and 2. Londres: Cambridge University Press, 1977.

MARIANO, V. C. A estrutura dos DPs em posição de sujeito no português rural afrodescendente. In: Revista Inventário, 11ํㅡㄹ ed, 2012, p. 1-15. 
.DPs nus na posição de sujeito no português afro-brasileiro. 2018. 94 f. Dissertação. (Mestrado em Língua e Cultura) Instituto de Letras, Salvador: UFBA.

MEDEIROS JÚNIOR, S. A. Análise das relativas restritivas locativas introduzidas por "onde que", no Português Brasileiro. 2017. 150 f. Dissertação. (Mestrado em Linguística) UESB, Vitória da Conquista.

MÓIA, T. Semântica e Pragmática. In: MARTINS, A. M.; CARRILLO, E (eds.). Manual de Linguística Portuguesa. Berlin/Boston: De Gruyter. 2016. p. 308-335.

MÜLLER, A. A expressão da genericidade nas línguas naturais. Relatório de Pós-doutorado. Massachussets: University of Massachussets-Amerst, 2000.

OLIVEIRA, R. P. O. Semântica Formal: uma breve introdução. Campinas: Mercado das Letras, 2001.

OLIVEIRA, R. P. O.; MEZARI, M. P. Os vários modos de ser nu: uma introdução. In: OLIVEIRA, R. P. O.; MEZARI, M. P. (Orgs.). Nominais nus: um olhar através das línguas. Campinas, SP: Mercado das Letras, 2012, p. 7-26.

PARTEE, B. Opacity, coreference, and pronouns. In:DAVIDSON, D.; HINTIKKA, J. (eds.). Word and Objections: Essays on the work of W. V. Quine. Dordrecht: Reidel, 1972, p. 415-441.

. Opacity, coreference, and pronouns. In: PARTEE, B. (ed.). Compositionaly in Formal Semantics. Malden: Blackwell, 2004, p. 26-49.

POSTAL, P. On so-called 'pronouns' in English. In.: DINNEEN, F. ed. 19 th Monograph on Languages and Linguistics. Washington, D.C.: Georgetown University Press, 1966.

RIBEIRO, I..; CYRINO, S. A expressão de DPs em dois registros de afro-brasileiros do século XIX. Apresentado no workshop "Gramaticalização: abordagens formais e funcionais", 2010.

ROSS, J. R. Constraints on variables in syntax. 1967. 523 f. Tese. (Doutorado em Filosofia) Departamento de Linguística e Línguas Modernas, MIT, Cambridge.

SCHIMITT C.; MUNN, A. Against the nominal mapping parameter: bare nouns in Brazilian Portuguese. Proceedings of NELS 29, 1999.

SARAIVA, M. E. F. O comportamento gramatical do SN nu objeto. In: . Buscar menino no colégio: a questão do objeto incorporado em português. Campinas: Pontes, 1997, p. 25-59.

SEDRINS, A. P. Restrições de extração de argumentos e adjuntos de nome no português brasileiro, 2009. 216f. Tese. (Doutorado em Linguística) UFAL, Maceió.

STRAWSON, P. F. On referring. In.: Mind, n.59, 1950, p. 320-344.

\section{OS AUTORES E O PPGLinC}

\section{Fernanda de Oliveira Cerqueira}

É Licenciada em Letras Vernáculas, na UFBA. Mestre e Doutora em Língua e Cultura, na antiga linha de Variação de Língua Portuguesa e Teoria da Gramática, pelo PPGLinC, na UFBA, com bolsa FAPESB (2015-2019), sob orientação do Prof. Dr. 
Danniel Carvalho, com quem lidera o grupo PHINA (A sintaxe-phi das línguas naturais). Desenvolveu estágio doutoral no Centro de Linguística, da Faculdade de Ciências Sociais e Humanas, da Universidade Nova de Lisboa, com bolsa Capes (2017). Foi representante estudantil no Colegiado do PPGLinC (2015-2016) e na Congregação do ILUFBA (2016-2017), atuando nas comissões de bolsa, de prêmio de tese, de reestruturação do programa e eleitoral. Foi docente substituta de Linguística, no ILUFBA (2019-2020). No momento, atua na educação básica, na rede privada. Seus anos no PPGLinC foram de muito aprendizado e aperfeiçoamento profissional, social e político, fundamentais para sua trajetória.

\section{Victor Cavalcanti Mariano}

Sou egresso do curso de Mestrado do PPGLinC da UFBA. No Programa, defendi, sob orientação do Professor Dr. Danniel Carvalho, a dissertação intitulada "DPs nus na posição de sujeito no português afro-brasileiro", no ano de 2018, pela linha de Variação de Língua Portuguesa e Teoria da Gramática. Nesse período, participei de disciplinas e atividades que me fizeram amadurecer como pesquisador e profissional, com professores que são modelos para a minha docência. Hoje, sou professor do Instituto Federal da Paraíba (IFPB), em que leciono as disciplinas de Língua Portuguesa e Literatura Brasileira para os cursos Técnicos Integrados ao Ensino Médio e de Metodologia Científica para os cursos superiores. Também sou tutor da disciplina Fundamentos da Linguística Românica no curso EAD de Letras. Além disso, sou um dos líderes do grupo de pesquisa Metodologias Ativas para o Ensino de Línguas (MAEL).

\section{A IMPORTÂNCIA DO PPGLinC PARA NOSSA FORMAÇÃO}

Para nós, o PPGLinC foi fundamental, uma vez que nos possibilitou experiências únicas de vivência acadêmica e aprendizagem. As disciplinas nos fizeram desvendar novos horizontes que nos abriram portas para o ensino e para pesquisa. Nesse sentindo, os professores deram uma contribuição ímpar, guiando-nos nos diversos caminhos teóricos e metodológicos, além de servirem como inspiração para nossa prática pedagógica. Além disso, a presteza dos funcionários do Instituto, como um todo, facilitou muito nossos caminhos ao longo da jornada na pós-graduação. Desse amplo rol de profissionais que contribuíram na nossa formação, gostaríamos de destacar o Prof. Dr. Danniel Carvalho, por ter nos orientado em nossas pesquisas ao longo da nossa formação e pela atenção e compreensão sempre disponíveis. Sem dúvidas, Danniel é um presente que o PPGLinC nos deu. Fica aqui nosso agradecimento ao Programa e todos que o fazem possível.

Nota do editor:

Artigo submetido para avaliação em: 05 de outubro de 2020.

Aprovado em sistema duplo cego em: 09 de março de 2021. 